\title{
Unique Ferroelectricity and Structure of Nylon 39 Induced by an Electric Field
}

\author{
Yasuhiro Matsuda ${ }^{* 1, \#}$, Osamu Achiwa ${ }^{* 2}$, Masato Inagaki ${ }^{* 1}$, Hidekazu Hirosawa $^{* 1}$, and Shigeru Tasaka $^{* 1,2}$ \\ ${ }^{* 1}$ Faculty of Engineering, Shizuoka University, Hamamatsu 432-8561, Japan \\ ${ }^{* 2}$ Graduate School of Science and Engineering, Shizuoka University, Hamamatsu 432-8561, Japan
}

\begin{abstract}
The structure of nylon 39 in the presence of an electric field was investigated by infrared spectroscopy. The vibration peak of the $\mathrm{N}-\mathrm{H}$ groups in nylon 39 in the presence of direct and alternative current shifted to a higher wavenumber, which was also observed in the infrared spectra of nylon 39 at higher temperatures. A very large dielectric constant for nylon $39\left(2000\right.$ at $\left.180^{\circ} \mathrm{C}\right)$ and remanent polarization were revealed by the dielectric relaxation spectra and displacement-electric hysteresis loops, respectively. These dielectric properties cannot be explained only by the arrangement of the dipole moments of $\mathrm{N}-\mathrm{H}$ in nylon 39. The change in the infrared spectra and unique electric properties can be explained by the partial dissociation of the hydrogen bonds and the ionization of the amide groups in nylon 39.

(Received 24 July, 2012 ; Accepted 8 December, 2012)
\end{abstract}

\section{Introduction}

The amide groups of nylon chains form hydrogen bonds that dominate their crystal structure and physical properties, especially their electric properties. The amide groups in nylons synthesized by the ring opening polymerization of lactams with odd numbers of carbon atoms are arranged such that these groups can form hydrogen bonds between polymer chains, and the resulting distorted structures lead to unique electric characteristics, such as pyroelectricity and piezoelectricity. [1-10]

Nylons synthesized by the polycondensation of dicarbonic acids with odd numbers of carbon atoms and diamines composed of odd-numbered carbon atoms have ferroelectricity because of their structures formed due to the hydrogen bonds between polymer chains. Scheinbeim et al. [11-15] detected the ferroelectricity of nylon 7 prepared by quenching and drawing at low temperature. Nylon 7 is forced to form distorted structures to form hydrogen bonds [3], and the samples with disordered structures prepared by quenching can be made ferroelectric by applying an electric field. Nylon 77 and fluoro-polyamides also showed ferroelectric behaviors in newly cast films. [16]

A very large dielectric constant $(\sim 1150)$ was observed in the dielectric relaxation spectra of nylon 1010 by $\mathrm{Lu}$ el al. [15]. They proposed that this behavior was caused by the accumulation of electric charge at the

\# corresponding author interface with the electrodes, a phenomenon known as Maxwell-Wagner-Sillars polarization.

In this communication, we focus on the change in the physical properties of nylon 39 induced by the application of an electric field. Because nylon 39, like nylon 7 and 77, is forced to adopt distorted conformations to form hydrogen bonds between two nylon 39 chains, the hydrogen bonds of nylon 39 are expected to be weak and changeable by applied electric fields. [3] Infrared spectra, dielectric relaxation spectra, and displacement-electric hysteresis loops were obtained to analyze the change in the hydrogen bond structure and the electric properties induced by the application of an electric field.

\section{Experimental}

Nylon 39 powder was prepared by the interfacial polycondensation of 1,3-diaminopropane and azelaic chloride. Differential scanning calorimetry for this sample was carried out, and melting temperature was determined to be $210^{\circ} \mathrm{C}$. No clear signal for glass transition could be detected.

The powder was made into thick films with thicknesses of micrometers with a spatula on a hotplate heated at about $225^{\circ} \mathrm{C}$. Thin films of nylon 39 were also prepared for transmission infrared spectroscopy in the presence of an applied electric field. Nylon 39 was dissolved in formic acid, and $1 \mu \mathrm{l}$ of the solution (1 $\mathrm{wt} \%)$ was dropped onto a silicon wafer onto which a gold electrode had been evaporated. After the formic acid was removed by heating the wafer at $180^{\circ} \mathrm{C}$, gold was 
evaporated again on the nylon 39 film to cross the gold electrode.

The transmission infrared spectra of nylon 39 in the presence of an electric field were measured for the thin films with electrodes prepared as described above. Infrared spectra were measured for nylon 39 film in the presence of both direct and alternative $(60 \mathrm{~Hz})$ current

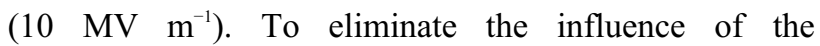
arrangement of nylon39 chains induced by direct current, a direct current of $12 \mathrm{MV} \mathrm{m}^{-1}$ was applied to the film before the measurements. Direct current with various voltages was applied again to the film during the measurements of infrared spectra. The direction of the infrared rays was fixed to the direction of the applied electric field. Fig. 1 illustrates a sample for infrared spectroscopy in the presence of an electric field.

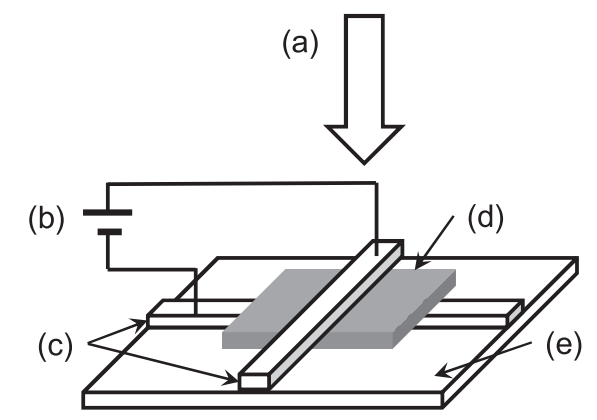

Fig. 1 A sample of nylon 39 for infrared spectroscopy in the presence of an electric field. (a) irradiation of infrared ray (b) power supplier (c) gold electrodes (d) nylon 39 (e) silicon wafer.

Reflective infrared spectra of nylon 39 heated at various temperatures were carried out for the thick films on a ceramic heater. Both transmission and reflective infrared spectra were obtained with a JASCO FI-IR 3000. We focused on the N-H vibration peak to analyze the structure and stability of the hydrogen bonds of nylon 39 . No significant signals caused by the presence of water in the samples were detected in the spectra obtained in this study.

A thick film was put on a glass plate onto which aluminum was evaporated. An aluminum layer was evaporated onto the nylon 39 film to cross the aluminum electrode. Dielectric measurements were performed in vacuo with an HP4284A type LCR meter (HewlettPackard) by heating at $3{ }^{\circ} \mathrm{C} / \mathrm{min}$.

Displacement-electric loops for the nylon 39 films with aluminum electrodes were obtained by applying a triangular electric field pulse with a frequency of $0.02 \mathrm{~Hz}$ using a wide-band function generator (Model FG-163, NF) and a voltage amplifier. The samples were prepared by the same method as dielectric measurements. The current density versus electric field curves obtained by subtracting the direct current conducting component according to the general process described in ref. 11 , and displacement-electric loops were calculated by integrating the current density versus electric field curves. The measurements were carried out at least 20 times to investigate the reproducibility, and the obtained hysteresis loops were identical to each other within experimental error.

\section{Results and discussion}

The peaks caused by N-H vibration in the infrared spectra of nylon 39 in the presence of alternative and direct electric currents at room temperature (below the glass transition temperature) are shown in Fig. 2. The spectra were normalized to the peak of $\mathrm{C}-\mathrm{H}$ vibration mode (ca. $2924 \mathrm{~cm}^{-1}$ ). The peaks of nylon 39 at 51 and $104^{\circ} \mathrm{C}$ in the absence of electric fields are also illustrated for comparison. The peak decreased and shifted to higher wavenumbers at higher temperatures, indicating that the hydrogen bonds between the nylon 39 chains became weaker at higher temperatures. The peaks of the infrared spectra of nylon 39 in the presence of alternative and direct electric fields also decreased and shifted to higher wavenumbers at higher voltages. The peak recovered the original shape after the electric field was removed. These results suggest that the electric field weakened the hydrogen bonds and changed the crystal structure of nylon 39. The dependence of the peak absorbance $A_{\text {peak }}$, and wavenumber $v_{\text {peak }}$ of a peak observed at ca. $3290 \mathrm{~cm}^{-1}$ on (a) the alternative electric field $E$, and (b) temperature $T$ is summarized in Fig. 3.

Fig. 4 shows the temperature dependence of the real part of the dielectric constant $\varepsilon$ ' and the loss tangent $\tan \delta$ of nylon 39. A small relaxation caused by the $\alpha$ relaxation mode, and a large dielectric constant $(>2000)$ were observed from the $\varepsilon$ ' of nylon 39. This dielectric behavior was observed in samples with high crystallinity. Similar large dielectric constants were also reported for nylon 46 and nylon1010. [15] These relaxations can be explained by Maxwell-Wagner-Sillars relaxation at the interfacial phases.

Displacement-electric hysteresis loops of nylon 39 at 80 and $130^{\circ} \mathrm{C}$ are shown in Fig. 5. The remanent polarization $P_{\mathrm{r}}$ was $40 \mathrm{mC} \mathrm{m}^{-2}$ at $80^{\circ} \mathrm{C}$, and $213 \mathrm{mC} \mathrm{m}^{-2}$ at $130^{\circ} \mathrm{C}$. If all of the $\mathrm{N}-\mathrm{H}$ dipoles in nylon 39 are completely arranged, the $P_{\mathrm{r}}$ can be calculated using the following equation, 


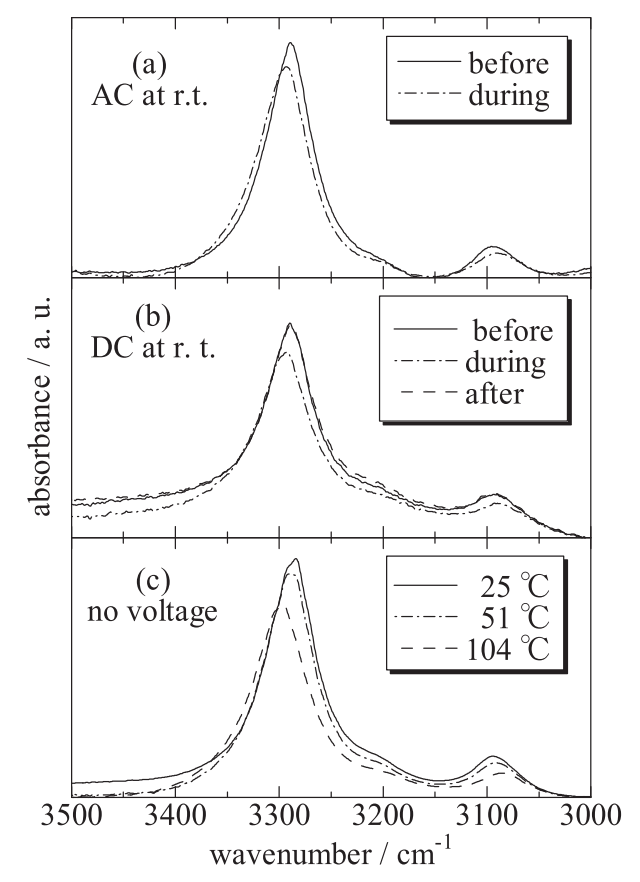

Fig. 2 Infrared peak of N-H vibration of nylon 39 before, during, and after applying (a) an alternative, and (b) a direct current electric field at room temperature. The peak of nylon 39 at various temperatures in the absence of an electric field (c) is also shown in the figure. The applied electric field and frequency for alternative current were $10 \mathrm{MV} \mathrm{m}^{-1}$ and $60 \mathrm{~Hz}$, respectively.

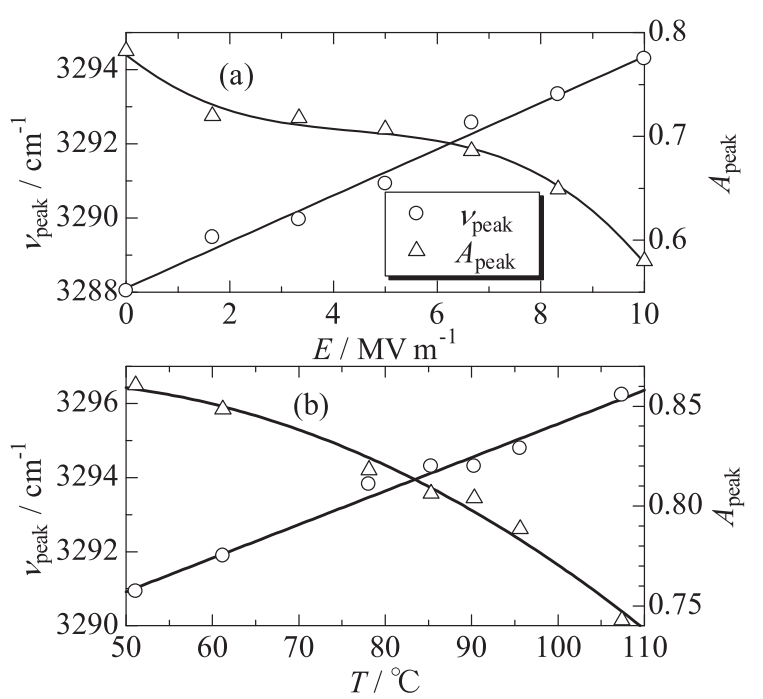

Fig. 3 Temperature dependence of the absorbance and wavenumber of a peak observed at ca. 3290 $\mathrm{cm}^{-1}$. on (a) the applied voltage and (b) temperature.

$$
P_{\mathrm{r}}=\frac{2 \mu \rho N_{\mathrm{A}}}{M_{0}}
$$

where $\mu, \rho, N_{\mathrm{A}}$, and $M_{0}$ repsresent the dipole moment of an amide group $\left(1.2 \times 10^{-29} \mathrm{C} \mathrm{m}\right)$, the density of nylon 39 $\left(1.11 \mathrm{~g} \mathrm{~cm}^{-3}\right)$, Avogadro's number $\left(6.02 \times 10^{23} \mathrm{~mol}^{-1}\right)$, and

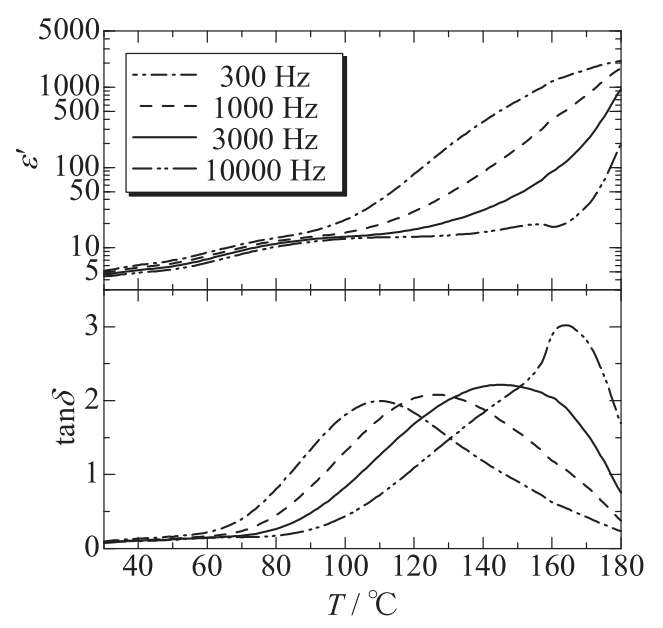

Fig. 4 Temperature dependence of $\varepsilon^{\prime}$ and $\tan \delta$ of nylon 39.

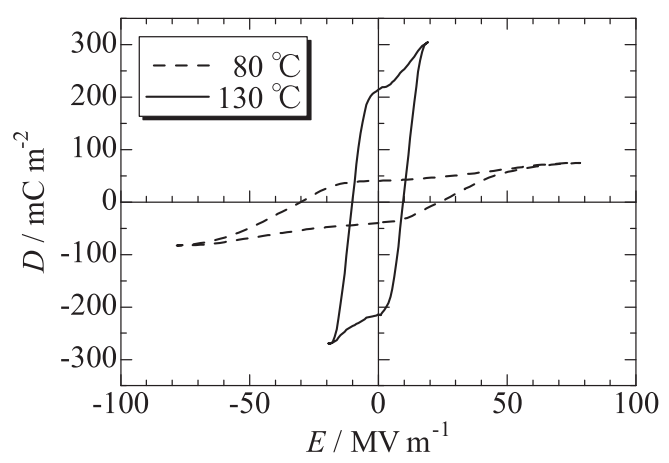

Fig. 5 Displacement-electric hysteresis loops of nylon 39.

the molecular weight of the repeating unit of nylon 39 $\left(226 \mathrm{~g} \mathrm{~mol}^{-1}\right)$, respectively.

Because $P_{\mathrm{r}}$ in the crystal region calculated from Eq. (1) is $71 \mathrm{mC} \mathrm{m}^{-2}$, the high values of $P_{\mathrm{r}}$ in Fig. 3 cannot be explained only by the arrangement of $\mathrm{N}-\mathrm{H}$ groups in the crystalline region of nylon 39. A large value of $P_{\mathrm{r}}$ is sometimes caused by ionic impurities in samples, but the reproducibility of the hysteresis loops observed in Fig. 5 rules out this possibility.

Based on the results described above, it is proposed that the applied electric field ionizes some amide groups of nylon 39 and weakens the hydrogen bonds, as illustrated in Fig. 6. The shift in the N-H vibration peak observed in the infrared spectra measured in the presence of applied electric fields supports the hypothesis that the hydrogen bonds are weak, as shown in Fig. 6. The very large $P_{\mathrm{r}}$ calculated from the displacement-electric hysteresis loops and the reproducibility of these loops can be explained by the rotation of the amide groups produced by partial ionization because the ionized amide groups have much larger dipole moments. 
without electric field or low temeprature

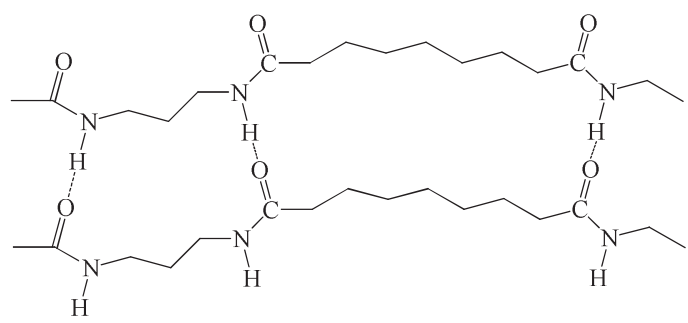

under electric field or high temeprature

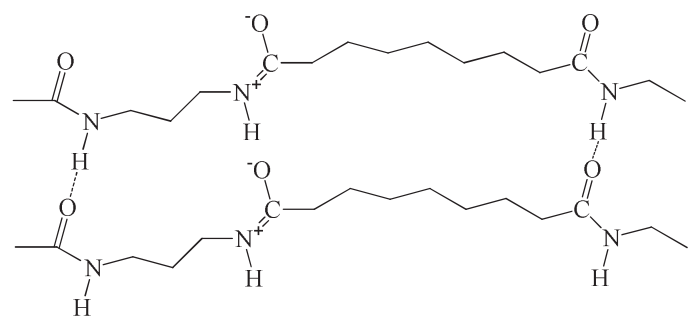

Fig. 6 Schematic images of nylon 39 chains at (a) low temperature or in the absence of an electric field and at (b) high temperature or in the presence of an electric field.

\section{Conclusion}

The hydrogen bond structure of nylon 39 induced by electric fields was investigated using infrared spectroscopy, dielectric measurements, and displacementelectric hysteresis loops. The N-H peak of the nylon 39 films shifted to a higher wavenumber in the presence of electric field like the N-H peak of the heated nylon 39 films. The dielectric properties cannot be explained only by the arrangement of the amide groups. A model that the applied electric field weakened the hydrogen bonds between the amide groups and ionized the amide groups was proposed to explain these experimental data.

\section{Acknowledgement}

This work is partially supported by a Grant-in Aid for Young Scientist (B) (23700869) from the Ministry of
Education, Culture, Science, Sports and Technology of Japan.

\section{References}

1. D. D. Coffman, G. J. Berchet, W. R. Peterson, and E. W. Spanagel, J. Polym. Sci. 2, 306 (1947).

2. R. Hill, and E. E. Walker, J. Polym. Sci. 3, 609 (1948).

3. Y. Kinoshita, Makromol. Chem. 33, 1 (1959).

4. Y. Kinoshita, Makromol. Chem. 33, 21 (1959).

5. M. H. Litt, C. H. Hsu, and P. Basu, J. Appl. Phys. 48, 2208 (1977).

6. M. H. Litt, and J. C. Lin, Ferroelectrics 48, 171 (1984)

7. B. A. Newman, P. Chen, K. D. Pae, and J. I. Sceinbeim, J. Appl. Phys. 51, 5161 (1980).

8. J. I. Sceinbeim, S. C. Mathur, and B. A. Newman : J. Polym. Sci. Part B Polym. Phys. 24, 1791 (1986).

9. S. C. Mathur, A. Sen, B. A. Newman, and J. I. Sceinbeim, J. Materials Sci. 23, 977 (1988).

10. S. C. Mathur, B. A. Newman, and J. I. Sceinbeim, J. Polym. Sci. Part B Polym. Phys. 26, 447 (1988).

11. J. W. Lee, Y. Takase, B. A. Newman, and J. I. Scheinbeim, J. Polym. Sci. Part B Polym. Phys. 29, 273 (1991).

12. J. W . Lee, Y. Takase, B. A. Newman, and J. I. Scheinbeim, J. Polym. Sci. Part B Polym. Phys. 29, 279 (1991).

13. Y. Takase, J. W. Lee, and J. I. Scheinbeim, Macromolecules 24, 6644 (1991).

14. J. I. Scheinbeim, J. W. Lee, and B. A. Newman, Macromolecules 25, 3729 (1992).

15. H. Lu, X. Zhang, and H. Zhang : J. Appl. Polym. Sci. 102, 3590 (2006).

16. A. C. Jayasuriya, S. Tasaka, M. Ohtani, and N. Inagaki, J. Appl. Phys. 79, 1017 (1996). 